\title{
Advances in Group-III-Nitride Photodetectors
}

\author{
Carlos Rivera*, ${ }^{*}$, Juan Pereiro ${ }^{1}$, Álvaro Navarro ${ }^{1}$, Elías Muñoz ${ }^{1}$, Oliver Brandt ${ }^{2}$ and \\ Holger T. Grahn ${ }^{2}$
}

\author{
${ }^{1}$ ISOM and Dpto. Ingeniería Electrónica, ETSI Telecomunicación, Universidad Politécnica de Madrid, Ciudad Univer- \\ sitaria, 28040 Madrid, Spain \\ ${ }^{2}$ Paul-Drude-Institut für Festkörperelektronik, Hausvogteiplatz 5-7, 10117 Berlin, Germany
}

\begin{abstract}
Group-III nitrides are considered to be a strategic technology for the development of ultraviolet photodetectors due to their remarkable properties in terms of spectral selectivity, radiation hardness, and noise. The potential advantages of these materials were initially obscured by their large density of intrinsic defects. The advances were thus associated in general with improvements in material quality. Although technology still also needs improvement, efforts are being intensified in the fabrication of advanced structures for photodetector applications. In particular, this review discusses the recent progress in group-III-nitride photodetectors, emphasizing the work reported on quantum-well-based photodetectors, the use of novel structures exploiting the effect of piezoelectric polarization-induced fields, and polarization-sensitive photodetectors. Furthermore, some ideas can be generalized to other material systems such as $\mathrm{ZnO}$ and their related compounds, which exhibit the same crystal structure as group-III nitrides.
\end{abstract}

Keywords: Photodetectors, Group-III nitrides, Quantum wells, Polarization-sensitive devices, Electrooptical modulation.

\section{INTRODUCTION}

The development of group-III nitrides in the late 1990's has been motivated by the realization of efficient light emitting diodes (LED), which are now an industrial standard for solid state lighting [1,2]. Other significant applications of group-III nitrides are high-frequency/high-power electronics using high-electron-mobility transistors (HEMT) and photodetectors $[3,4]$. Photodetectors based on group-III nitrides take advantage of their inherent spectral selectivity to allow for the tuning of the detection edge from about $200 \mathrm{~nm}$ (AlN) to about $1770 \mathrm{~nm}(\mathrm{InN})$. Some potential benefits added by the use of these materials are their radiation hardness and the expected lower intrinsic noise and dark current in comparison with narrow-band-gap materials (e.g., Si) [5, 6]. In addition, the devised applications comprise, for example, solar-blind detection $[7,8]$, non-line-of-sight ultraviolet (UV) communications [9], combustion monitoring [10], space communications [11, 12], high-resolution photolithography together with emitters [13], and missile plume detection [11]. Similarly, solar cells fabricated with $(\mathrm{In}, \mathrm{Ga}) \mathrm{N}$ compounds operating in the $2.4 \mathrm{eV}$ range, as photodetectorlike devices, are a promising technology to increase the conversion efficiency of current photovoltaic cells [14-16].

In this work, we describe the recent advances and the future prospects of group-III-nitride photodetectors. They include the detection in the visible (VIS) [17, 18], extremeUV (EUV), and soft x-ray ranges [19, 20], novel device designs and concepts such as gain mechanisms based on piezoelectricity or nanowire photodetectors $[21,22]$, new func-

*Address correspondence to this author at the ISOM and Dpto. Ingeniería Electrónica, ETSI Telecomunicación, Universidad Politécnica de Madrid, Ciudad Universitaria, 28040 Madrid, Spain; Tel: +34-91-549.57.00 Ext. 4208; Fax: +34-91-336.73.23; E-mail: crivera@ die.upm.es tionalities (e.g., polarization sensitivity or narrow-band detection) $[23,24]$, and new scenarios (e.g., high-temperature environments) [25]. This review is organized as follows. Section II presents a summary of the main parameters used in the photodetector characterization. The objective of this section is to help understand the decisions taken in the design of photodiodes. In section III, previous research on and the properties of group-III-nitride photodetectors is briefly discussed. The main body concerning recent advances in group-III-nitride photodetectors is divided into three sections, IV, V, and VI, which correspond to basic structures, multiple-quantum-well (MQW)-based photodiodes, and polarization-sensitive photodiodes, respectively. Finally, the conclusions are presented in section VII, where, in addition to the summary of the results, we propose new directions in the field of group-III-nitride photodetectors.

\section{USEFUL DEFINITIONS IN PHOTODETECTOR CHARACTERIZATION}

Photodetectors can be characterized by a set of parameters or figures of merit which are of interest in practical applications. In particular, we consider the following figures of merit: responsivity, noise, and speed. The importance of each figure of merit will depend on the application, and, in most cases, they are not independent, allowing for the existence of design rules constrained by simple tradeoff relations (e.g., the photoconductive gain increases at the expense of a reduction in the response time). A detailed understanding of the device structure is needed to determine the value of the figures of merit and, therefore, to be able to improve the performance of the photodetectors.

The responsivity is defined as the ratio of the electrical output to the optical input for a given wavelength and can be expressed as 
$R=\frac{I_{p h}}{P}$,

where $I_{p h}$ denotes the photocurrent and $P$ the incident optical power on the device. $I_{p h}$ depends in turn on the absorption and the current collection efficiency. The absorption is determined by the active layer thickness and the absorption coefficient for photons not reflected at the surface (the reflectance at normal incidence between air and $\mathrm{GaN}$ is about $15 \%$ below the absorption edge). The parameter used to characterize the absorption is known as the quantum efficiency. Assuming that all photogenerated carriers contribute to the photocurrent and no gain is present, the maximum responsivity is given by $e / h v$. This limit can be increased for photons with energies of at least twice the band gap energy so that more than one electron-hole pair per photon can be photogenerated [26-28]. Note that photogenerated carriers can either recombine before reaching the contacts or contribute to gain. The existence of gain mechanisms can be used to design devices with high responsivity. The most representative mechanisms are the photoconductive gain and the avalanche gain. The photoconductive gain in basic devices (i.e., photoconductors) is given by

$g=\frac{\tau_{v}}{t_{t r}}\left(1+\frac{\mu_{h}}{\mu_{e}}\right)$,

where $\tau_{v}$ is the minority carrier lifetime, $t_{t r}$ the majority carrier transit time, and $\mu_{e}\left(\mu_{h}\right)$ the electron mobility (hole mobility). Since, in general, $\mu_{e}>>\mu_{h}$, it follows that $g \approx \tau_{v} / t_{t r}$. Therefore, the gain-bandwidth product of the photoconductor depends only on $t_{t r}$ taking into account that the response time is limited by $\tau_{v}$. The second mechanism takes advantage of a high electric field to initiate an avalanche process driven by photogenerated carriers. The high electric field is reached by applying a high external voltage. In both cases, a price is usually paid in terms of an increase in the dark current, in addition to the already mentioned shortcomings (speed for the photoconductive gain and the need of a high applied voltage for the avalanche gain). These examples illustrate the tradeoff relations one faces in the design of photodetector structures. Group-III nitrides open the possibility to study new types of photoconductive gain as will be discussed below.

Other remarkable characteristics of the responsivity are related to its spectral and power dependence. The spectral selectivity is the most obvious advantage offered by groupIII-nitride semiconductors compared to other wellestablished semiconductors, which need filters to operate in the VIS and UV regions. The selectivity is typically defined by the detection band and the rejection ratio or contrast between the desired and undesired bands. The dependence of the responsivity as a function of power is assessed by comparing it with a linear response. Linear operation is preferred for most applications. However, high-gain photodetectors frequently exhibit a nonlinear response.

Noise is related to the sensitivity or minimum input power that can be detected. Thus, the sensitivity is defined as the optical power which generates an electrical signal equal to that due to noise. A quantity of interest to characterize the sensitivity is the noise equivalent power (NEP), which is defined as

$N E P=\frac{i_{n}}{R}$ where $i_{n}$ is the effective value of the noise current of the photodiode. In order to improve the performance of photodetectors, the noise must be minimized. Due to the close relation between noise and dark current, it is convenient to design photodiodes operating under low dark current conditions.

Finally, the speed is characterized by the bandwidth $(B W)$ of the frequency response. The bandwidth can be limited by intrinsic factors (e.g., minority carrier lifetime) or the external circuit ( $R C$ limited). All these figures of merit can be combined into a common parameter for the comparison of photodetectors known as the specific detectivity, which is given by

$D^{*}=\frac{\sqrt{A_{o p} B W}}{N E P}$,

where $A_{o p}$ is the effective area for detection. Note that a higher bandwidth increases both the speed and the noise of the photodetector. In general, the higher $D^{*}$ is, the better the photodetector. However, there are other factors such as selectivity or linear dependence as a function of power that can be more critical for certain applications.

\section{BACKGROUND}

The basic properties of wurtzite nitrides are summarized in Fig. (1). We can observe that the detection of VIS and IR light requires the use of (In,Ga)N or (In,Al,Ga)N alloys with high In mole fractions, whereas the detection of deep UV light requires the use of $(\mathrm{Al}, \mathrm{Ga}) \mathrm{N}$ or $(\mathrm{In}, \mathrm{Al}, \mathrm{Ga}) \mathrm{N}$ alloys with high Al mole fractions. The different properties of these compounds have determined the further development of this technology, as it will be discussed later. The inset of Fig. (1) also shows the valence band (VB) structure for unstrained $\mathrm{GaN}$ including the spin-orbit interaction [29]. The effect of strain on the wave functional character and energies of the VB states will be discussed in Section VI.

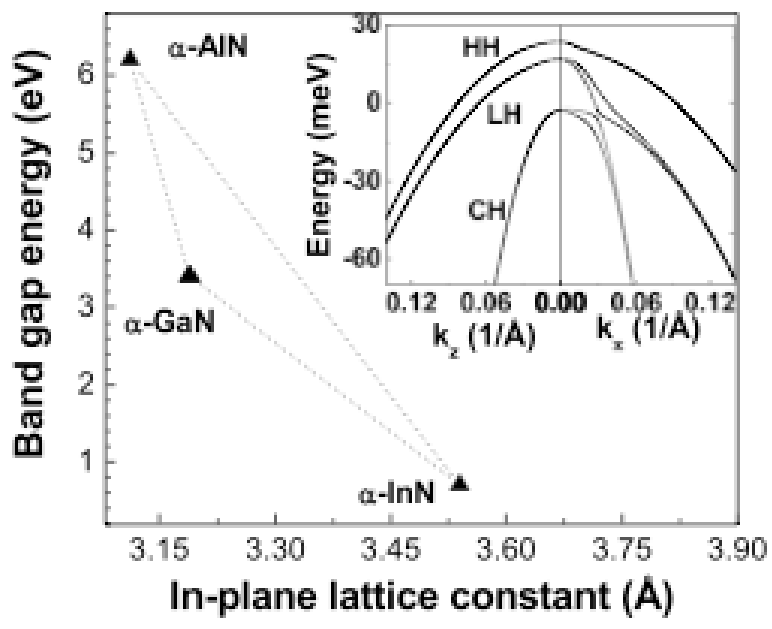

Fig. (1). Band gap energy and in-plane lattice constant for wurtzite (A1,In,Ga)N compounds. Inset: Valence band structure for unstrained wurtzite $\mathrm{GaN}$ including the spin-orbit interaction (the effective mass parameter $A_{7}=93.7 \mathrm{meV} / \AA$ [29]).

Now focusing on devices, the work carried out in groupIII-nitride photodetectors can be classified into several stages. Initially, research was focused on the fabrication of simple structures such as $(\mathrm{Al}, \mathrm{Ga}) \mathrm{N}$ photoconductors and Schottky barrier photodiodes grown by metalorganic chemi- 
cal vapor deposition (MOCVD) or molecular beam epitaxy (MBE). The objective was to study the effect of the material quality on the basic properties of the devices. The main characteristic offered by these photodetectors was the spectral selectivity, mainly for solar-blind applications [30-35].

Later, with the advances achieved in material quality, innovations in the design of photodetectors to improve their performance in terms of spectral selectivity, noise, responsivity, and speed were introduced. Some illustrative examples can be found in Refs. [36, 37]. In this period, specific detectivities higher than $10^{13} \mathrm{cmHz}^{1 / 2} \mathrm{~W}^{-1}$, which are similar to those of commercial $\mathrm{Si}$ diodes, and response times of about 1 ps were reported.

Currently, the research focuses on different lines related to device optimization, array or charge-coupled-device fabrication, and avalanche photodiodes [38-40]. Most work has been performed to develop photodetectors operating in the UV region. In contrast, there are only a few studies extending the detection range to the VIS and infrared (IR) regions, basically for technological reasons [17].

\section{BASIC STRUCTURES}

The current research on device optimization consists of improving material quality and contact technology. Illustrative examples are the use of double buffer structures [41], unconventional nucleation layers [42], and new materials for contacts to increase the detection range [43] or even the specific detectivity [44]. The results of Ref. [41] show that radiation hardness can be enhanced by decreasing the defect density. Similarly, the improvement in material quality allows for the reduction in the dark current and the increase in the barrier height in Schottky barrier photodiodes [42]. In Refs. [43, 44], contacts made of TiW and Ir/Pt were used, respectively. TiW contacts exhibit a higher transmittance at higher energies (<300 $\mathrm{nm}$ range) than $\mathrm{Ni} / \mathrm{Au}$. However, $\mathrm{Ir} / \mathrm{Pt}$ contact technology is beneficial to reduce the dark current due to the higher barrier height. Other important examples of research related to device optimization can be found in Refs. [45-47].

The interest of increasing the sensitivity linked to the advances in material quality has motivated the study of avalanche photodetectors [48]. Promising results have been reported by different groups for $(\mathrm{Al}, \mathrm{Ga}) \mathrm{N}$ [49], GaN [50], and AlN [51]. Namely, reproducible gain values of about $10^{3}$ have been achieved using (Al,Ga)N [49], whereas values as high as $3 \times 10^{3}$ have been reached with $\mathrm{GaN}$ [38]. Nevertheless, there is still room for improvement, since, for example, the size of the reported devices is small (below $0.002 \mathrm{~mm}^{2}$ ). Another approach to obtain high sensitivity is based on quantum wires. These structures intrinsically exhibit a large photoconductive gain, as also found for $\mathrm{ZnO}[52,53]$. The origin of the large photoconductive gain seems to be related to surface trapping along the current transport direction.

In the last few years, novel design concepts have also been developed including dual-band or multiple-band detection $[54,55]$ and quantum-well-based photodetectors (cf. Section V). These designs aim at an improved spectral selectivity and sensitivity. Fig. (2) shows a good example of a device design to enhance the sensitivity by reducing the dark current, whereas the responsivity is barely enhanced. In this case, the device is a simple photoconductor consisting of an
(In,Ga)N/GaN/(In,Ga)N $n-i-n$ structure. The basic idea to reduce the dark current is to introduce a $\mathrm{GaN}$ barrier between the $(\mathrm{In}, \mathrm{Ga}) \mathrm{N}$ active layers. The photocurrent increases for vertical transport in comparison to lateral transport since $t_{t r}$ decreases. Therefore, we can design an optimized photoconductor in terms of dark current and responsivity using a vertical geometry and a current blocking layer. Results related to the anisotropy in detectivity for vertical and lateral structures have also been reported in Ref. [56]. In addition, the built-in potential induced by piezoelectric fields allows for a nonzero photoresponse under photovoltaic operation.

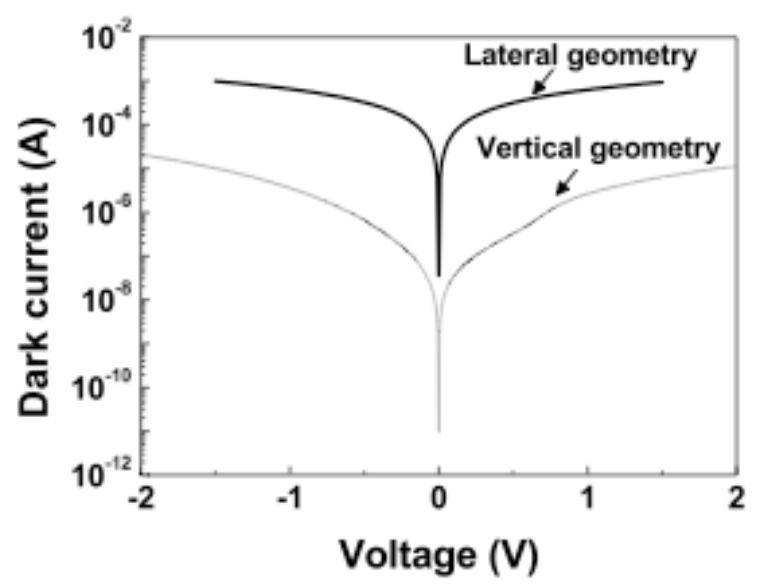

Fig. (2). Dark current versus voltage for an $n-i-n$ structure consisting of (In,Ga)N/GaN/(In,Ga)N using a lateral (upper curve) and a vertical geometry (lower curve).

Other interesting photodetectors are those based on metal-insulator structures (MIS). These structures are designed to reduce the leakage current and, therefore, the dark current, of Schottky barrier photodetectors, while maintaining similar responsivity values. Some examples of photodetectors taking advantage of the MIS structures can be found in Refs. [57-59].

For applications, there is growing interest in the realization of photodetectors operating in the deep UV [60, 61]. Most works report on the characterization of $(\mathrm{Al}, \mathrm{Ga}) \mathrm{N}$ photodetectors with high Al content using simple structures such as metal-semiconductor-metal (MSM) and Schottky barrier photodiodes, due to the difficulties in both $n$ - and $p$-type doping. Note that a photodetector based on cubic BN for the deep UV region has been recently reported [62]. This material offers interesting properties for optoelectronic and acoustic applications [63]. Alternatively, the interest in the VIS and IR ranges has motivated the fabrication of (In,Ga)N-based photodiodes [17, 64-66]. These devices exhibit low internal quantum efficiencies $(<16 \%)$ and poor electrical performance. The reasons for the poor performance in comparison with $\mathrm{GaN}$-based devices are again deficiencies in the material and contact quality. In the next section, MQW-based photodetectors are discussed as an alternative to photodetection in the VIS region.

\section{5. (IN,GA)N/GAN MULTIPLE-QUANTUM-WELL- BASED PHOTODIODES}

The use of (In,Ga)N/GaN MQW structures was initially proposed to overcome the technological limitations imposed by $(\mathrm{In}, \mathrm{Ga}) \mathrm{N}$-bulk technology in terms of reproducibility and 
electrical characteristics (e.g., dark current) [67-70]. Despite the deficiencies in the material and contact quality, these structures offer remarkable advantages such as a lower expected noise, a more abrupt photoresponse edge, an easier integration of emitter and detector functions, an extra flexibility to design the device characteristics, and the possibility to generate new gain mechanisms [18, 21, 71]. Regarding applications, these devices have shown promising results for biological analysis, combustion monitoring, and solar energy conversion $[72,73]$.

Fig. (3) shows the photoresponse at zero bias of Schottky diodes fabricated on three samples S1, S2, and S3 with a 5period (In, Ga) N/(In, Al,Ga)N MQW active region, where the In content is $7 \%, 6 \%$, and $2 \%$, respectively. Well and barrier thicknesses are approximately 3 and $17 \mathrm{~nm}$, respectively, for all three samples (S1, S2, and S3). In Fig. (3), we can distinguish two spectral regions corresponding to the absorption in the QWs (long wavelengths) and the barriers (short wavelengths). Note that the barriers for samples S1, S2, and S3 are $\mathrm{GaN}, \mathrm{In}_{0.02} \mathrm{Ga}_{0.98} \mathrm{~N}$, and $\mathrm{Al}_{0.16} \mathrm{Ga}_{0.84} \mathrm{~N}$, respectively. The lower responsivity of QW photodiodes reflects the lower absorption compared to that of the barriers. Nevertheless, the efficiency is close to unity, i.e., each absorbed photon in the QWs contributes to the photocurrent with one electron-hole pair. Recent studies indicate the same trend for a larger number of QWs, but only when the QWs are located in the space charge region [18]. Thus, we expect in this case that increasing the number of QWs leads to a higher responsivity, eventually reaching the responsivity of bulk material. Moreover, the absorption edge is more abrupt than the typical one found in $(\mathrm{In}, \mathrm{Ga}) \mathrm{N}$ bulk for the same In content. Recent publications serve as a proof of concept to show that MQW-based structures for above band gap photodetection are a solid alternative to standard detectors $[18,67-70]$. For example, the values of $D^{*}$ are typically around $10^{12} \mathrm{cmHz}^{1 / 2} \mathrm{~W}^{-1}$, which are comparable to the best values reported for bulk-based devices operating under similar conditions.

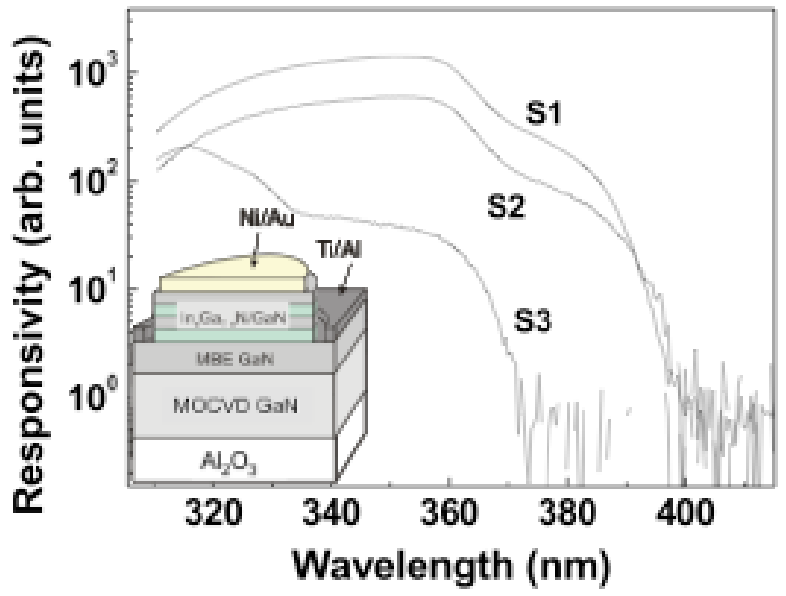

Fig. (3). Spectral responsivity for three different (In,Ga)N/GaN MQW-based Schottky barrier photodetectors S1, S2, and S3 (see description in the text) with different composition in wells and barriers. Inset: Device structure.

An intrinsic, although not exclusive, advantage of MQWbased photodetectors is the possibility to obtain a bandpass response for energies between the barrier and QW energy gaps. In Fig. (4), the GaN substrate acts as an optical filter for back illumination. The bandpass photoresponse of these (In,Ga)N/GaN MQW-based $p-i-n$ photodetectors can thus be tuned by changing the In content. In this case, backilluminated photodiodes with an In content of 10\%, 19\%, and $25 \%$ have been used. Note that the contrast between the peak responsivity and the responsivities for the undesired bands is better than $10^{3}$.

Group-III-nitride-based MQW structures can also be exploited to design another class of nonlinear optical devices through the electrical modulation of the optical properties. As polar heterostructures, they offer the possibility to operate in a highly nonlinear optical regime based on the photogenerated-carrier-induced screening of the built-in electric field without applying a bias voltage. The key point is that in polar heterostructures the built-in electric field can intrinsically remove the inversion symmetry in quantum wells resulting in a nonzero first-order Stark effect. The larger the optical nonlinearity is, the better the performance. For these reasons, polar heterostructures are attractive for their use in optical switching and logic devices.

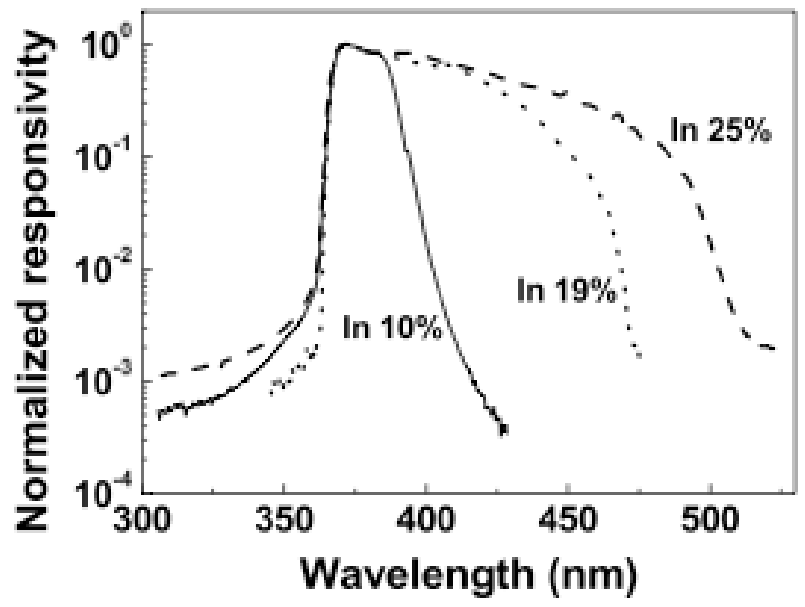

Fig. (4). Normalized spectral responsivity for three backilluminated (In,Ga)N/GaN MQW-based photodiodes with an In content of $10 \%, 19 \%$, and $25 \%$.

In the case of the $(\mathrm{In}, \mathrm{Ga}) \mathrm{N} / \mathrm{GaN}$ system, QWs are subjected to a high built-in electric field $F$ [74]. The effective band gap energy in this type of structures, assuming that $F$ is large, can be estimated as

$E_{g}^{e f f}=E_{g}+E_{q}-e F L$,

with $E_{g}$ being the band gap energy, $E_{q}$ the confinement energy, $e$ the electronic charge, $F$ the electric field, and $L$ the well thickness. According to Eq. (5), the main energy shift is thus related to the potential-energy difference induced by the electric field across the well. The confinement energy also changes. Again, assuming a large electric field, we can treat the problem as that of a triangular QW so that the groundstate energy can be written as

$E_{q}=\left(\frac{9 \pi e \hbar F}{8 \sqrt{2}}\right)^{2 / 3}\left(\frac{1}{m_{e}}+\frac{1}{m_{h}}\right)^{1 / 3}$,

where $m_{e}$ and $m_{h}$ are the effective masses for the conduction (CB) and VBs, respectively, and $\hbar$ is the reduced Planck constant. Note that under these conditions the energy shift in Eq. (5) is a slightly sub-linear function of $F$ as expected by the 
the nonzero value of the first-order correction calculated using perturbation theory. Fig. (5) shows the change in the differential responsivity as a function of applied voltage for a $p-i$ - $n$ photodetector based on (In,Ga)N/GaN QWs [18]. The differential responsivity is approximately proportional to the absorption coefficient. We can observe that, in contrast to standard electroabsorption modulators, the energy shift in absorption occurs towards higher energies with increasing applied voltage. This result is reasonable taking into account that the external voltage in this case reduces the pre-existing built-in electric field, hence decreasing the Stark shift. A higher In content leads to a higher built-in electric field.

Similarly to the modulation of the optical properties, we can induce a modulation of the electrical properties in the MQW structure. In this case, we apply the reverse concept, i.e., light is used to modify the electrical properties. This modification can in turn be exploited to detect light or increase the photodetection capabilities. Of the many possibilities of modulation, we will discuss the one arising from the change in the internal electric field, namely, in the built-in electric field induced by the piezoelectric polarization. The same idea can be employed for other types of built-in electric fields, but this modulation takes advantage of the utilization of MQW-based structures.

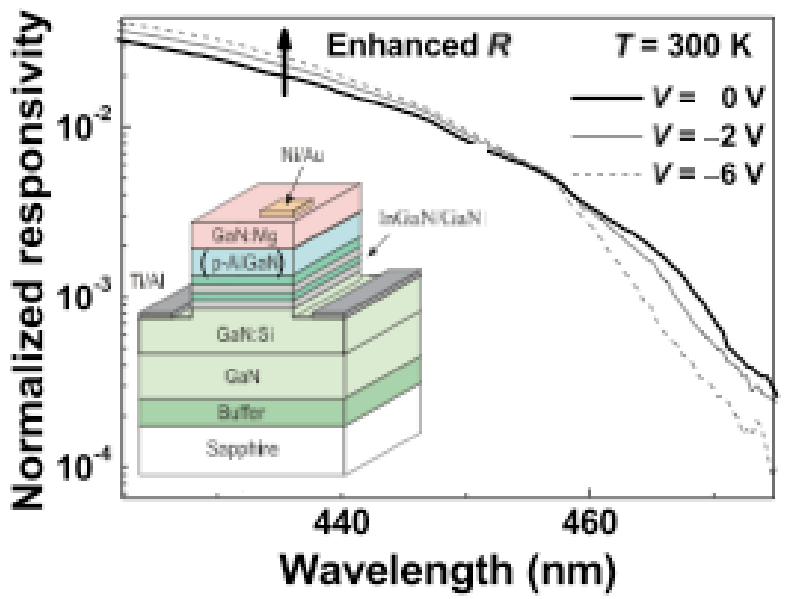

Fig. (5). Normalized spectral responsivity for a 3-period $($ In,Ga)N/GaN MQW-based $p-i-n$ photodiode at different applied voltages. Inset: Device structure.

The interplay of polarization-induced electric fields and other built-in electric fields (e.g., the electric field in a $p-n$ junction) can result in a tunable envelope potential. An interesting case appears when the average electric field including polarization-induced effects is opposite to the built-in electric field present in the structure without any polarizationinduced effects. This situation, which can be found in heterostructures based on polar materials, is called negative average electric field (NAF) [75, 76]. Structures designed to work under NAF conditions have been proposed to improve the performance of photodetectors by means of internal photocurrent gain [21]. In this case, the average electric field in the active region can be modulated by both an external applied voltage and optical excitation. The applied voltage determines the operation conditions in terms of speed and dark current in the linear regime of low optical excitation levels. In contrast, optical modulation can be comparable to electrical modulation under high optical excitation so that the over- all device characteristic is affected. These properties may result in new opportunities for the design of advanced photodetectors. Fig. (6) illustrates the case of a Schottky barrier structure similar to that of S1, except that the barriers are unintentionally doped. Here, we can observe that the NAF condition is reached when the potential drop in the barriers is lower than the potential drop of opposite sign in the QWs. The positive applied voltage will decrease the electric field in the barriers, allowing the generation of an NAF region. The opposite situation will be reached by applying a negative bias, tending to create a region with a positive average electric field. It can be observed that the NAF region acts as a barrier for transport. For already small positive voltages, the barrier generated by the NAF domain becomes higher than the barrier imposed by the metal-semiconductor interface as shown in Fig. (6) at $1 \mathrm{~V}$. The typical band bending in the space charge region is recovered at negative voltages. Note that polarization-induced field effects are strongly quenched in highly doped samples since free carriers screen the resultant electric fields. Therefore, the existence of an NAF region is difficult to detect, or it is not even present anymore under certain conditions. Other factors such as defects or the barrier height are also important for determining the role of polarization-induced field effects on the device performance. Experimental results confirmed that a high gain could be achieved under NAF conditions for a device with the same nominal structure used for the calculations of Fig. (6). Internal fields can also be tailored to generate the suitable band diagram to develop quantum-cascade photodetectors, as it has been reported in Ref. [77].

Finally, group-III-nitride MQW-based photodetectors can exhibit a giant photocurrent gain of photoconductive nature [78] similar to other materials. The mechanisms involved in the photocurrent gain have not been identified yet, though possible explanations are related to trapping or different transit times for electrons and holes in the superlattice (this last mechanism is known as effective mass filtering) [79].

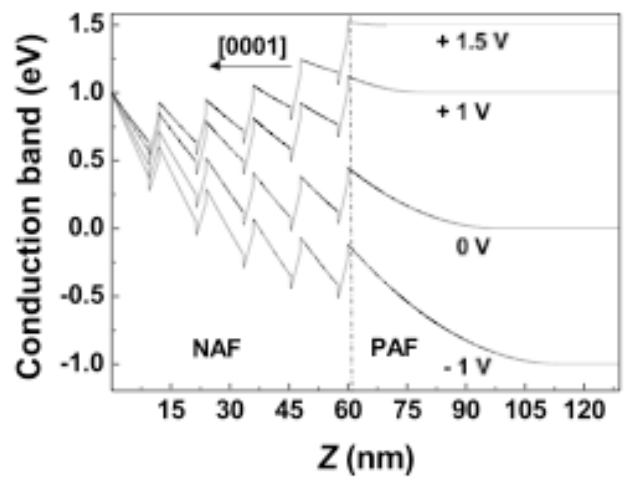

Fig. (6). Conduction band diagram of an (In,Ga)N/GaN MQWbased Schottky barrier photodetector at different applied voltages. The vertical line separates the region where a negative average electric field can be formed (at $+1 \mathrm{~V}$ and $+1.5 \mathrm{~V}$ in the figure) from the region of positive average electric field.

\section{POLARIZATION-SENSITIVE PHOTODETECTORS}

More recently, the use of the intrinsic in-plane polarization anisotropy for nonpolar-oriented materials such as $M$ plane or $A$-plane $\mathrm{GaN}$ enhanced by the effect of anisotropic strain has been proposed to fabricate polarization-sensitive 
photodetectors (PSPDs) [80]. These photodetectors can be used in several applications including data storage, optical communications, natural object recognition, marine biology, biophotonics, and computer vision, among others [81]. The change in the crystal orientation from the $C$ plane to the $M$ plane produces a modification of the selection rules for incident light perpendicular and parallel to the in-plane direction in such a way that the transition between the uppermost VB and the CB becomes linearly polarized perpendicular to the $c$ axis $(\mathbf{E} \perp \mathbf{c})$. Moreover, the reduction of the in-plane crystal symmetry can be further enhanced by the anisotropic strain generated by the lattice mismatch between the nitride film and the substrate, which can result in an almost complete linear polarization of the transition between the $\mathrm{CB}$ and the second uppermost VB state parallel to the $c$ axis $(\mathbf{E} \| \mathbf{c})$ and of the transition between the $\mathrm{CB}$ and the third uppermost VB state with $\mathbf{E} \perp$ c. Note that the same results are also applicable to the $A$ plane since the Hamiltonian is symmetric with respect to the interchange of the corresponding directions describing the $M$ and $A$ planes.

Fig. (7) shows the photoresponse of a typical $M$-plane $\mathrm{GaN}$ photodetector for different in-plane polarization angles $\varphi$ under normal incidence. We can clearly observe that the photodetection edge is blueshifted as the polarization of the incoming light changes from $\mathbf{E} \perp \mathbf{c}$ to $\mathbf{E} \| \mathbf{c}$. In general, the angular dependence of responsivity can be expressed as

$R=R_{\perp} \sin ^{2}(\varphi)+R_{\|} \cos ^{2}(\varphi)$,

where $R_{\perp}$ and $R_{\|}$are the components of the responsivity for $\mathbf{E}$ $\perp \mathbf{c}$ and $\mathbf{E} \| \mathbf{c}$, respectively. The dependence on $\varphi$ found in Eq. (7) is the main difference between the standard photodetector and the PSPD. The polarization sensitivity depends on the in-plane strain values and the total absorption of the film. On the one hand, the in-plane strain affects the VB splitting and, therefore, the region of the spectrum where the photoresponse is polarization sensitive. On the other hand, the ratio between the absorption for the different polarizations determines the maximum achievable contrast $R_{\perp} / R_{\|}$. Both of them depend in turn on the film thickness and the type of substrate. For a fixed substrate, the design of PSPDs must take into account the effect of the active film thickness in addition to other constraints [23].

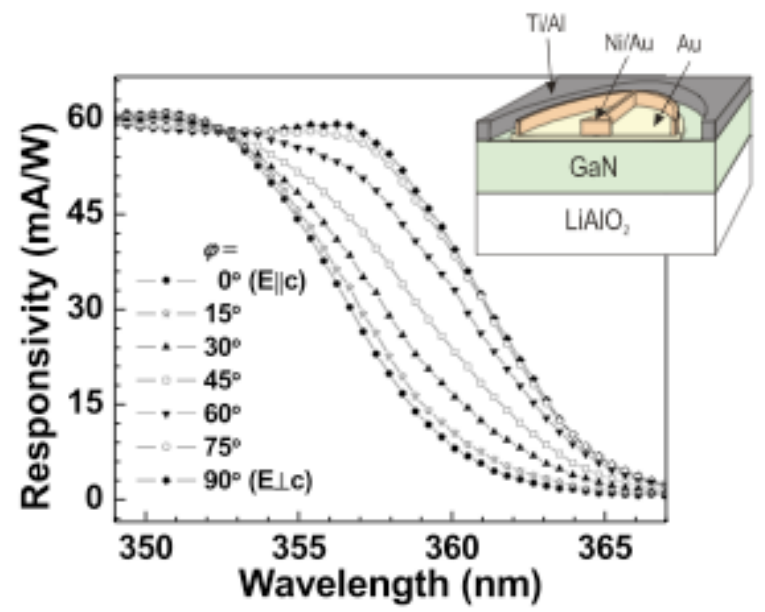

Fig. (7). Spectral responsivity for an $M$-plane GaN-based photodetector at different in-plane polarization angles under normal incidence. Inset: Device structure.
PSPDs based on $M$-plane GaN reported so far have been grown on $\gamma-\mathrm{LiAlO}_{2}$ substrates and realized as Schottky barrier or metal-insulator-semiconductor structures (MIS) [23, 82]. Schottky barrier devices exhibit high leakage currents and show the best performance under photovoltaic operation. In this case, responsivities are typically in the range of 60 $\mathrm{mA} / \mathrm{W}$. Other studies suggest that current leakage is related to surface conduction [83]. Although surface passivation using MIS photodiodes has been proven to alleviate the high leakage currents at reverse bias, the responsivity also decreased, resulting in similar or even lower detectivity values [82]. Recently, PSPDs using a metal-semiconductor-metal structure based on high-resistive $A$-plane $\mathrm{GaN}$ grown on sapphire substrates have also been reported [84]. These devices exhibit very low dark currents, leading to high photocurrent gain and detectivity values better than $10^{12}$ $\mathrm{cmHz}^{1 / 2} \mathrm{~W}^{-1}$ at high voltages. However, the optical anisotropy is smaller than the one measured for $M$-plane $\mathrm{GaN}$ due to the lower strain induced by the lattice mismatch between the film and the substrate.

In addition to polarization-sensitive applications, PSPDs can also be exploited for narrow-band detection as it was recently demonstrated utilizing either a configuration which combines two pieces of the same material, where one acts as a filter and the other as a detector, or a configuration consisting of two or four photodetectors, where each one is oriented at a certain angle with respect to a reference axis [24, 85]. Therefore, these PSPDs can be used for measuring the optical properties of light, namely, color (i.e., wavelength), intensity, polarization and spatial variation, opening the possibilities for a large variety of applications.

\section{CONCLUSIONS}

Photodetectors based on group-III nitrides are already commercially available for UV applications. However, there is still room to improve the material quality, although the advances in fabrication have been noteworthy. Note that, in addition for photodetectors, this need is shared by other more demanding technologies such as for HEMTs or LEDs.

Novel photodetectors taking advantage of the intrinsic properties of nitrides have been devised. On the one hand, the strong piezoelectricity present in these materials offers an additional design tool to modify the band diagram of the device structure, allowing for new photocurrent gain mechanisms and nonlinear properties as well as increasing photodetector capabilities. On the other hand, the reduction in the crystal symmetry, which is enhanced by the anisotropic strain, results in a strong optical anisotropy, which can be exploited to fabricate polarization-sensitive photodetectors. Furthermore, quantum effects have also been explored, providing promising results for MQW- and quantum-wire-based photodetectors. Future research lines should include spectrally selective photodetectors using PSPDs, integrated filters, photonic crystal structures, and the possibility to extend the photodetection range towards the visible and infrared spectral regions. The properties of $\mathrm{ZnO}$ compounds make these materials potential candidates for optoelectronic devices by taking advantage from the advances in nitrides (e.g., see Ref. [86] for a hybrid $\mathrm{GaN}-\mathrm{ZnO}$ photodetector). 


\section{REFERENCES}

[1] S. Nakamura, and G. Fasol, The Blue Laser Diode. Berlin, Springer, 1997.

[2] C. Wetzel, M. Zhu, J. Senawiratne, T. Detchprohm, P.D. Persans, L. Liu, E.A. Preble, and D. Hanser, "Light-emitting diode development on polar and non-polar GaN substrates", J. Cryst. Growth, vol. 310, pp. 3987-3991, 2008.

[3] U.K. Mishra, L. Shen, T.E. Kazior, and Y.F. Wu, "GaN-Based RF Power Devices and Amplifiers", Proc. IEEE, vol. 96, pp. 287-305, 2008.

[4] E. Muñoz, E. Monroy, J.L. Pau, F. Calle, F. Omnès, and P. Gibart, "III nitrides and UV detection", J. Phys.: Condens. Matter., vol. 13, pp. 7115-7137, 2001.

[5] M. Razeghi and A. Rogalski, "Semiconductor ultraviolet detectors", J. Appl. Phys., vol. 79, pp. 7433-7473, 1996.

[6] A. Ionascut-Nedelcescu, C. Carlone, A. Houdayer, H.J. von Bardeleben, J.-L. Cantin, and S. Raymond, "Radiation hardness of gallium nitride”, IEEE Trans. Nucl. Sci., vol. 49, pp. 2733-2738, 2002.

[7] D. Walker, V. Kumar, K. Mi, P. Sandvik, P. Kung, X.H. Zhang, and M. Razeghi, "Solar-blind AlGaN photodiodes with very low cutoff wavelength", Appl. Phys. Lett., vol. 76, pp. 403-405, 2000.

[8] B.W. Lim, Q.C. Chen, J.Y. Yang, and M. Asif Khan, "High responsitivity intrinsic photoconductors based on $\mathrm{Al}_{\mathrm{x}} \mathrm{Ga}_{1_{-\mathrm{x}} \mathrm{N}}$ ", Appl. Phys. Lett., vol. 68, pp. 3761-3762, 1996.

[9] G.A. Shaw, A.M. Siegel, and J. Model, "Ultraviolet comm link for distributed sensor systems", IEEE LEOS Newslett., vol. 19, pp. 2629, 2005.

[10] J. Schalwig, G. Müller, O. Ambacher, and M. Stutzmann, "Group III-nitride-based sensors for combustion monitoring”, Mater. Sci. Eng. B, vol. 93, pp. 207-214, 2002.

[11] A. Rogalski and M. Razeghi, "Semiconductor ultraviolet photodetectors", Opto-Electron. Rev., vol. 4, pp. 13-30, 1996.

[12] S.N. Mohammad, A. Salvador, and H. Morkoç, Emerging gallium nitride based devices. Proc. IEEE, vol. 83, 1995, pp. 1306-1355.

[13] F.A. Ponce and D.P. Bour, "Nitride-based semiconductors for blue and green light-emitting devices", Nature (Lond.), vol. 386, pp. 351-359, 1997.

[14] A. De Vos, Endoreversible Thermodynamics of Solar Energy Conversion. Oxford: Oxford University Press, 1992.

[15] J. Wu, W. Walukiewicz, K.M. Yu, W. Shan, J.M. Ager III, E.E. Haller, H. Lu, W.J. Schaff, W.K. Metzger, and S. Kurtz, "Superior radiation resistance of $\mathrm{In}_{1-x} \mathrm{Ga}_{x} \mathrm{~N}$ alloys: full-solar-spectrum photovoltaic material system", J. Appl. Phys., vol. 94, pp. 6477-6482, 2003.

[16] O. Jani, I. Ferguson, C. Honsberg, and S. Kurtz, "Design and characterization of GaN/InGaN solar cells", Appl. Phys. Lett., vol. 91, p. $132117,2007$.

[17] J.C. Roberts, C.A. Parker, J.F. Muth, S.F. Leboeuf, M.E. Aumer, S.M. Bedair, and M.J. Reed, "Ultraviolet-visible metalsemiconductor-metal photodetectors fabricated from $\operatorname{In}_{(\mathrm{x})} \mathrm{Ga}_{(1-\mathrm{x})} \mathrm{N}$ (0 < x < 13)", J. Electron. Mater., vol. 31, pp. 94-99, 2002.

[18] C. Rivera, J.L. Pau, A. Navarro, E. Muñoz, "Photoresponse of (In,Ga)N-GaN multiple-quantum-well structures in the visible and UVA ranges", IEEE J. Quant. Electron., vol. 42, pp. 51-58, 2006.

[19] A. Motogaito, K. Ohta, K. Hiramatsu, Y. Ohuchi, K. Tadatomo, Y. Hamamura, and K. Fukui, "Characterization of GaN based UVVUV detectors in the range $3.4-25 \mathrm{eV}$ by using synchrotron radiation", Phys. Stat. Sol. A, vol. 188, pp. 337-340, 2001.

[20] J.L. Pau, C. Rivera, E. Muñoz, E. Calleja, U. Schühle, E. Frayssinet, B. Beaumont, J.P. Faurie, and P. Gibart, "Response of ultralow dislocation density GaN photodetectors in the near- and vacuum-ultraviolet", J. Appl. Phys., vol. 95, pp. 8275-8279, 2004.

[21] C. Rivera, J.L. Pau, E. Muñoz, "Photocurrent gain mechanism in Schottky barrier photodiodes with negative average electric field", Appl. Phys. Lett., vol. 89, p. 263505, 2006.

[22] S. Han, W. Jin, D. Zhang, T. Tang, C. Li, X. Liu, Z. Liu, B. Lei, and C. Zhou, "Photoconduction studies on GaN nanowire transistors under UV and polarized UV illumination", Chem. Phys. Lett., vol. 389, pp. 176-180, 2004.

[23] C. Rivera, J.L. Pau, E. Muñoz, P. Misra, O. Brandt, H.T. Grahn, and K.H. Ploog, "Polarization-sensitive ultraviolet photodetectors based on $M$-plane GaN grown on $\mathrm{LiAlO}_{2}$ substrates", Appl. Phys. Lett., vol. 88, p. 213507, 2006.

[24] S. Ghosh, C. Rivera, J.L. Pau, E. Muñoz, O. Brandt, and H.T. Grahn, "Very narrow-band ultraviolet photodetection based on strained M-plane GaN films", Appl. Phys. Lett., vol. 90, 091110, 2007.

[25] A. Navarro, C. Rivera, R. Cuerdo, J.L. Pau, J. Pereiro, and E. Muñoz, "Low frequency noise in InGaN/GaN MQW based photodetector structures", Phys. Stat. Sol. A, vol. 204, pp. 262-266, 2007.

[26] C.A. Klein, "Bandgap dependence and related features of radiation ionization energies in semiconductors", J. Appl. Phys., vol. 39, pp. 2029-2038, 1968.

[27] F. Scholze, H. Rabus, and G. Ulm, "Mean energy required to produce an electron-hole pair in silicon for photons of energies between 50 and 1500 eV", J. Appl. Phys., vol. 84, pp. 2926-2939, 1998.

[28] F. Scholze, H. Henneken, P. Kuschnerus, H. Rabus, M. Richter, and G. Ulm, "Determination of the electron-hole pair creation energy for semiconductors from the spectral responsivity of photodiodes", Nucl. Inst. Method, vol. 439, pp. 208-215, 2000.

[29] G.B. Ren, Y.M. Liu, and P. Blood, "Valence-band structure of wurtzite GaN including the spin-orbit interaction", Appl. Phys. Lett., vol. 74, pp. 1117-1119, 1999.

[30] E. Muñoz, E. Monroy, J.A. Garrido, I. Izpura, F.J. Sánchez, M.A. Sánchez-García, E. Calleja, B. Beaumont, and P. Gibart, "Photoconductor gain mechanisms in GaN ultraviolet detectors", Appl. Phys. Lett., vol. 71, pp. 870-872, 1997.

[31] E. Monroy, F. Calle, E. Muñoz, F. Omnès, P. Gibart, and J.A. Muñoz, " $\mathrm{Al}_{\mathrm{x}} \mathrm{Ga}_{1-\mathrm{x}} \mathrm{N}: \mathrm{Si}$ Schottky barrier photodiodes with fast response and high detectivity", Appl. Phys. Lett., vol. 73, pp. 21462148, 1998.

[32] J.A. Garrido, E. Monroy, I. Izpura, and E. Muñoz, "Photoconductive gain modelling of GaN photodetectors", Semicond. Sci. Technol., vol. 13, pp. 563-568, 1998.

[33] D.J.H. Lambert, M.M. Wong, U. Chowdhury, C. Collins, T. Li, H.K. Kwon, B.S. Shelton, T.G. Zhu, J.C. Campbell, and R.D. Dupuis, "Back illuminated AlGaN solar-blind photodetectors", Appl. Phys. Lett., vol. 77, pp. 1900-1902, 2000.

[34] N. Biyikli, O. Aytur, I. Kimukin, T. Tut, and E. Ozbay, "Solarblind AlGaN-based Schottky photodiodes with low noise and high detectivity", Appl. Phys. Lett., vol. 81, pp. 3272-3274, 2002.

[35] R. McClintock, A. Yasan, K. Mayes, D. Shiell, S.R. Darvish, P. Kung, and M. Razeghi, "High quantum efficiency AlGaN solarblind p-i-n photodiodes", Appl. Phys. Lett., vol. 84, pp. 1248-1250, 2004.

[36] M. Yonemaru, A. Kikuchi, and K. Kishino, "Improved responsivity of AlGaN-based resonant cavity-enhanced UV photodetectors grown on sapphire by RF-MBE", Phys. Stat. Sol. A, vol. 192, pp. 292-295, 2002.

[37] T. Tut, N. Biyikli, I. Kimukin, T. Kartaloglu, O. Aytur, M.S. Unlu, and E. Ozbay, "High bandwidth-efficiency solar-blind AlGaN Schottky photodiodes with low dark current", Solid State Electron., vol. 49, pp. 117-122, 2005.

[38] J.B. Limb, D. Yoo, J.H. Ryou, W. Lee, S.C. Shen, R.D. Dupuis, M.L. Reed, C.J. Collins, M. Wraback, D. Hanser, E. Preble, N.M. Williams, and K. Evans, "GaN ultraviolet avalanche photodiodes with optical gain greater than 1000 grown on GaN substrates by metal-organic chemical vapor deposition", Appl. Phys. Lett., vol. 89, p. $011112,2006$.

[39] R. McClintock, J.L. Pau, K. Minder, C. Bayram, P. Kung, and M. Razeghi, "Hole initiated multiplication in back-illuminated GaN avalanche photodiodes", Appl. Phys. Lett., vol. 90, p. 141112, 2007.

[40] J.P. Long, S. Varadaraajan, J. Matthews, and J.F. Schetzina, "UV detectors and focal plane array imagers based on AlGaN p-i-n photodiodes", Opto-Electron. Rev., vol. 10, pp. 251-260, 2002.

[41] H.F. Lui, W.K. Fong, and C. Surya, "Characteristics of MBEgrown GaN detectors on double buffer layers under high-power ultraviolet optical irradiation", IEEE Trans. Electron. Devices, vol. 54, pp. 671-676, 2007.

[42] Y.D. Jhou, S.J. Chang, Y.K. Su, Y.Y. Lee, C.H. Liu, and H.C. Lee, "GaN Schottky barrier photodetectors with SiN/GaN nucleation layer", Appl. Phys. Lett., vol. 91, p. 103506, 2007.

[43] C.K. Wang, S.J. Chang, Y.K. Su, Y.Z. Chiou, S.C. Chen, C.S Chang, T.K. Lin, H.L. Liu, and J.J. Tang, "GaN MSM UV Photodetectors With TitaniumTungsten Transparent Electrodes", IEEE Trans. Electron. Devices, vol. 53, pp. 38-42, 2006.

[44] C.L. Yu, C.H. Chen, S.J. Chang, and P.C. Chang, "GaN metalsemiconductor-metal ultraviolet photodetectors with $\mathrm{Ir} / \mathrm{Pt}$ contact electrodes", J. Electrochem. Soc., vol. 154, pp. J71-J72, 2007. 
[45] H.F. Lui, W.K. Fong, C. Surya, C.H. Cheung, and A.B. Djuri, "Fabrication and characterization of indium-tin-oxide/GaN visibleblind UV detectors", Phys. Stat. Sol. C, vol. 3, pp. 2295-2298, 2005.

[46] S. Butun, M. Gökkavas, H. Yu, and E. Ozbay, "Low dark current metal-semiconductor-metal photodiodes based on semi-insulating GaN", Appl. Phys. Lett., vol. 89, p. 073503, 2006.

[47] K.H. Lee, S.J. Chang, P.C. Chang, Y.C. Wang, and C.H. Kuo, "High quality GaN-based Schottky barrier diodes", Appl. Phys. Lett., vol. 93, p. 132110, 2008.

[48] Y. Wang, K.F. Brennan, and P.P. Ruden, "Theoretical study of a potential ultraviolet avalanching detector based on impact ionization out of confined quantum states", IEEE J. Quantum Electron., vol. 27, pp. 232-237, 1991.

[49] T. Tut, M. Gokkavas, A. Inal, and E. Ozbay, " $\mathrm{Al}_{\mathrm{x}} \mathrm{Ga}_{1-\mathrm{x}} \mathrm{N}$-based avalanche photodiodes with high reproducible avalanche gain", Appl. Phys. Lett., vol. 90, p. 163506, 2007.

[50] J.C. Carrano, D.J.H. Lambert, C.J. Eiting, C.J. Collins, T. Li, S. Wang, B. Yang, A.L. Beck, R.D. Dupuis, and J.C. Campbell, "GaN avalanche photodiodes", Appl. Phys. Lett., vol. 76, pp. 924-926, 2000.

[51] R. Dahal, T.M. Al Tahtamouni, J.Y. Lin, and H.X. Jiang, "AlN avalanche photodetectors", Appl. Phys. Lett., vol. 91, p. 243503, 2007.

[52] H. Kind, H.Q. Yan, B. Messer, M. Law, and P.D. Yang, "Nanowire Ultraviolet Photodetectors and Optical Switches", Adv. Mater., vol. 14, pp. 158-160, 2002.

[53] Z. Fan, P.C. Chang, J.G. Lu, E. Walter, R.M. Penner, C.H. Lin, and H.P. Lee, "Photoluminescence and polarized photodetection of single ZnO nanowires", Appl. Phys. Lett., vol. 85, pp. 6128-6130, 2004.

[54] G. Ariyawansa, M.B.M. Rinzan, M. Alevli, M. Strassburg, N. Dietz, A.G.U. Perera, S.G. Matsik, A. Asghar, I.T. Ferguson, H. Luo, A. Bezinger, and H.C. Liu, "GaN/AlGaN ultraviolet/infrared dual-band detector”, Appl. Phys. Lett., vol. 89, p. 091113, 2006.

[55] L.D. Bell, N. Tripathi, J.R. Grandusky, V. Jindal, and F.S. Shahedipour-Sandvik, "III-Nitride Heterostructure Layered Tunnel Barriers For a Tunable Hyperspectral Detector", IEEE Sens. J., vol. 8, pp. 724-729, 2008.

[56] O. Katz, V. Garber, B. Meyler, G. Bahir, and J. Salzman, "Anisotropy in detectivity of GaN Schottky ultraviolet detectors: Comparing lateral and vertical geometry", Appl. Phys. Lett., vol. 80, pp. 347-349, 2002.

[57] G.H. Yang, J.D. Hwang, C.H. Lan, C.M. Chan, H.Z. Chen, and S.J. Chang, "Indium-tin-oxide metal-insulator-semiconductor $\mathrm{GaN}$ ultraviolet photodetectors using liquid-phase-deposition oxide", Jpn. J. Appl. Phys., vol. 46, pp. 5119-5121, 2007.

[58] J.D. Hwang and C.J. Lin, "High 366/254-nm Rejection Contrast GaN MIS Photodetectors Using Nano Spin-Oxide", IEEE Electron Device Lett., vol. 30, p. $2729,2009$.

[59] P.C. Chang, C.H. Chen, S.J. Chang, Y.K. Su, C.L. Yu, B.R. Huang, and P.C. Chen, "High UV/visible rejection contrast AlGaN/GaN MIS photodetectors", Thin Solid Films, vol. 498, pp. 133-136, 2006.

[60] S. Aslam, L. Miko, C. Stahle, D. Franz, D. Pugel, B. Guan, J.P. Zhang, and R. Gaska, "Dual-band deep ultraviolet AlGaN photodetectors", Electron. Lett., vol. 43, pp. 1382-1384, 2007.

[61] G. Mazzeo, J.L. Reverchon, G. Conte, A. Dussaigne, and J.Y. Duboz, "Dynamics of AlGaN based detectors in the deep-UV", Solid State Electron., vol. 52, pp. 795-800, 2008.

[62] A. Soltani, H.A. Barkad, M. Mattalah, B. Benbakhti, J.C. De Jaeger, Y.M. Chong, Y.S. Zou, W.J. Zhang, S.T. Lee, A. BenMoussa, B. Giordanengo, and J.F. Hochedez, "193 nm deep-ultraviolet solar-blind cubic boron nitride based photodetectors", Appl. Phys. Lett., vol. 92, p. 053501, 2008.

[63] Ü. Özgür, Y.I. Alivov, C. Liu, A. Teke, M.A. Reshchikov, S. Dogan, V. Avrutin, S.J. Cho, and H. Morkoç, "A comprehensive review of ZnO materials and devices", J. Appl. Phys., vol. 98, p. 041301, 2005

[64] Y.K. Su, Y.Z. Chiou, F.S. Juang, S.J. Chang, and J.K. Sheu, "GaN and InGaN metal-semiconductor-metal photodetectors with different schottky contact metals", Jpn. J. Appl. Phys., vol. 40, pp. 29962999, 2001.

[65] J.J. Zhou, B. Wen, R.L. Jiang, C.X. Liu, X.L. Ji, Z.L. Xie, D.J. Chen, P. Han, R. Zhang, and Y.D. Zheng, "Photoresponse of the
$\mathrm{In}_{0.3} \mathrm{Ga}_{0.7} \mathrm{~N}$ metal-insulator-semiconductor photodetectors", Chin. Phys., vol. 16, pp. 2120-2123, 2007.

[66] E.A. Berkman, N.A. El-Masry, A. Emara, and S.M. Bedair, "Nearly lattice-matched n, i, and p layers for InGaN p-i-n photodiodes in the 365-500 nm spectral range", Appl. Phys. Lett., vol. 92, p. $101118,2008$.

[67] Y.Z. Chiou, Y.K. Su, S.J. Chang, J. Gong, Y.C. Lin, S.H. Liu, and C.S. Chang, "High detectivity InGaN-GaN multiquantum well p-n junction photodiodes", IEEE J. Quantum Electron., vol. 39, pp. 681-685, 2003.

[68] C. Rivera, J.L. Pau, F.B. Naranjo, and E. Muñoz, "Novel photodetectors based on InGaN/GaN multiple quantum wells", Phys. Stat. Sol. A, vol. 201, pp. 2658-2662, 2004.

[69] C. Rivera, J.L. Pau, J. Pereiro, and E. Muñoz, "Properties of schottky barrier photodiodes based on InGaN-GaN MQW structures", Superlatt. Microstruct., vol. 36, pp. 849-857, 2004.

[70] P.C. Chang, C.L. Yu, S.J. Chang, K.H. Lee, C.H. Liu, and S.L. Wu, "High-detectivity nitride-based MSM photodetectors on InGaN$\mathrm{GaN}$ multiquantum well with the unactivated Mg-doped $\mathrm{GaN}$ layer", IEEE J. Quantum Electron., vol. 43, pp. 1060-1064, 2007.

[71] C. Rivera, "Photodetectors based on quantum-well structures: theory, properties and novel concepts" A. Ruyter and H. O'Mahoney, Eds., in Quantum Wells: Theory, Fabrication and Applications, Nova Publishers, 2009, pp. 1-47.

[72] J.L. Pau, J. Anduaga, C. Rivera, A. Navarro, I. Álava, M. Redondo, and E. Muñoz, "Optical sensors based on III-nitride photodetectors for flame sensing and combustion monitoring", Appl. Opt., vol. 45, pp. 7498-7503, 2006.

[73] O. Jani, C. Honsberg, A. Asghar, D. Nicol, I. Ferguson, A. Doolittle, and S. Kurtz, "Characterization and analysis of InGaN photovoltaic devices", in Proc. $31^{s t}$ IEEE Photovolt. Specialists Conf., 2005, vol. 10, pp. 37-42.

[74] F. Bernardini, V. Fiorentini, and D. Vanderbilt, "Spontaneous polarization and piezoelectric constants of III-V nitrides", Phys. Rev. B, vol. 56, pp. R10024-R10027, 1997.

[75] A.S. Pabla, J.L. Sánchez-Rojas, J. Woodhead, R. Grey, J.P.R. David, G.J. Rees, G. Hill, M.A. Pate, P.N. Robson, R.A. Hogg, T.A. Fischer, R.K. Willcox, D.M. Whittaker, M.S. Skolnick, "Tailoring of internal fields in InGaAs/GaAs multiwell structures grown on (111)B GaAs", Appl. Phys. Lett., vol. 63, pp. 752-754, 1993.

[76] J.L. Sánchez-Rojas, A. Sacedón, F. Calle, E. Calleja, and E. Muñoz, "Conduction-band engineering in piezoelectric [111] multiple quantum well p-i-n photodiodes", Appl. Phys. Lett., vol. 65, pp. 2214-2216, 1994.

[77] A. Vardi, G. Bahir, F. Guillot, C. Bougerol, E. Monroy, and S.E. Schacham, "Near infrared quantum cascade detector in GaN/AlGaN/AlN heterostructures", Appl. Phys. Lett., vol. 92, p. 011112, 2008.

[78] C. Rivera and E. Muñoz, "Observation of giant photocurrent gain in highly doped (In,Ga)N/GaN MQW-based photodiodes", Appl. Phys. Lett., vol. 92, 233510, 2008.

[79] F. Capasso, K. Mohammed, A.Y. Cho, R. Hull, and A.L. Hutchinson, "New quantum photoconductivity and large photocurrent gain by effective-mass filtering in a forward-biased superlattice p-n junction", Phys. Rev. Lett., vol. 55, pp. 1152-1155, 1985.

[80] S. Ghosh, O. Brandt, H.T. Grahn, and K.H. Ploog, "Strained $M$ plane GaN for the realization of polarization-sensitive photodetectors", Appl. Phys. Lett., vol. 81, pp. 3380-3382, 2002.

[81] L.B. Wolff and T.E. Boult, "Constraining object features using a polarization reflectance model", IEEE Trans. Pattern Anal. Mach. Intell., vol. 13, pp. 635-657, 1991.

[82] C. Rivera, J.L. Pau, E. Muñoz, P. Misra, O. Brandt, H.T. Grahn, and K.H. Ploog, " $M$-plane GaN-based dichroic photodetectors", Phys. Stat. Sol. C, vol. 4, pp. 86-89, 2007.

[83] C. Rivera, J.L. Pau, E. Muñoz, P. Misra, O. Brandt, H.T. Grahn, and K.H. Ploog, "Electrical and optical characterization of $M$-plane GaN films grown on $\mathrm{LiAlO}_{2}$ substrates", Phys. Stat. Sol. C, vol. 4 , pp. 2548-2551, 2007.

[84] A. Navarro, C. Rivera, J. Pereiro, E. Muñoz, B. Imer, S.P. DenBaars, J.S. Speck, "High responsivity A-plane GaN-based metalsemiconductor-metal photodetectors for polarization-sensitive applications", Phys. Lett. 2009, (in press).

[85] C. Rivera, E. Muñoz, O. Brandt, and H.T. Grahn, "Detection of the optical polarization angle with bandpass characteristics based on 
M-plane GaN photodetectors", Appl. Phys. Lett., vol. 91, p. 203514, 2007.

[86] M.L. Lee, P.F. Chi, and J.K. Sheu, "Photodetectors formed by an indium tin oxide/zinc oxide/p-type gallium nitride heterojunction with high ultraviolet-to-visible rejection ratio", Appl. Phys. Lett., vol. 94, p. 013512, 2009

Received: April 15, 2009

Revised: October 27, 2009

Accepted: November 13, 2009

(C) Rivera et al.; Licensee Bentham Open.

This is an open access article licensed under the terms of the Creative Commons Attribution Non-Commercial License (http://creativecommons.org/licenses/by-nc/3.0/) which permits unrestricted, non-commercial use, distribution and reproduction in any medium, provided the work is properly cited. 